\title{
NOISE EFFECTS IN THE QUANTUM SEARCH ALGORITHM FROM THE VIEWPOINT OF COMPUTATIONAL COMPLEXITY
}

\author{
PIOTR GAWRON，JERZY KLAMKA，RYSZARD WINIARCZYK \\ Institute of Theoretical and Applied Informatics \\ Polish Academy of Sciences, ul. Bałtycka 5, 44-100 Gliwice, Poland \\ e-mail: gawron@itis.pl
}

\begin{abstract}
We analyse the resilience of the quantum search algorithm in the presence of quantum noise modelled as trace preserving completely positive maps. We study the influence of noise on the computational complexity of the quantum search algorithm. We show that it is only for small amounts of noise that the quantum search algorithm is still more efficient than any classical algorithm.
\end{abstract}

Keywords: quantum algorithms, quantum noise, algorithm complexity.

\section{Introduction}

It is often said that the strength of quantum computation lies in the phenomena of quantum superposition and quantum entanglement. These features of quantum computation allow performing the computation on all possible inputs that fit the quantum register. One of the greatest achievements in the theory of quantum algorithms is the quantum search algorithm introduced by Grover. A detailed description of this algorithm can be found in the works of Grover (1996; 1997; 1998) and Bugajski (2001).

Any physical implementation of a quantum computer will be error-prone because of the interaction of the computing device with the environment. In this paper we investigate the resilience of Grover's algorithm in the presence of quantum noise. We use the language of density matrices and quantum channels. Our goal is to find the maximal amount of noise for which the quantum algorithm is better, in terms of the mean number of operations, than the classical algorithm. We aim to achieve this objective by considering some classes of quantum channels modelling environmentally induced noise.

The paper is organised as follows. In Section 2 we provide a short review of the subject. In Section 3 we describe the formalism of quantum information theory. In Section 4 we present the quantum search algorithm. In Section 5 we introduce the noise model we have applied to the system. In Section 6 we analyse the results, and finally in Section 7 we present some conclusions.

\section{Review of existing work}

The problem of the influence of noise on the quantum search algorithm has been extensively studied by various researchers. Barnes and Warren (1999) discuss the influence of the classical field upon a quantum system implementing Grover's algorithm. Pablo-Norman and Ruiz-Altaba (1999) pose a question similar to ours, but use a Gaussian noise model, which in their case is not described in the language of quantum channels. Long et al. (2000) analyse how imperfections in realizations of quantum gates influence the probability of the success of the quantum search algorithm. Konstadakis and Ellinas (2001) analyse the behaviour of the quantum search algorithm implemented with the use of noisy $\pi / 4$ rotation gates.

The effect of unitary noise on the quantum search algorithm is studied by Shapira et al. (2003). Shenvi et al. (2003) examine the robustness of Grover's search algorithm to a random phase error in the oracle and analyse the complexity of the search process. Azuma (2005) studies decoherence in Grover's quantum search algorithm using a perturbation method. Zhirov and Shepelyansky (2006) use the methods of quantum trajectories to study the effects of dissipative decoherence on the accuracy of Grover's quantum search algorithm. Salas (2008) numerically simulates Grover's algorithm introducing random errors of two types: one- and two-qubit gate errors and memory ones. 


\section{Formalism of quantum information}

3.1. Dirac notation. Throughout this paper we use the Dirac notation. The symbol $|\psi\rangle$ denotes a complex column vector, $\langle\psi|$ denotes the row vector dual to $|\psi\rangle$. The scalar product of vectors $|\psi\rangle,|\phi\rangle$ is denoted by $\langle\psi \mid \phi\rangle$. The outer product of these vectors is denoted by $|\phi\rangle\langle\psi|$. Vectors are labelled in a natural way: $|0\rangle:=\left(\begin{array}{l}1 \\ 0\end{array}\right),|1\rangle:=\left(\begin{array}{l}0 \\ 1\end{array}\right)$. Notation such as $|\phi \psi\rangle$ denotes the tensor product of vectors and is equivalent to $|\phi\rangle \otimes|\psi\rangle$.

3.2. Density operators. The most general state of a quantum system is described by a density operator. In quantum mechanics a density operator $\rho$ is defined as a Hermitian $\left(\rho=\rho^{\dagger}\right)$ positive semi-definite $(\rho \geq 0)$ trace one $(\operatorname{tr}(\rho)=1)$ operator. When a basis is fixed, the density operator can be written in the form of a matrix. Diagonal density matrices can be identified with probability distributions, and therefore this formalism is a natural extension of probability theory.

Density operators are usually called quantum states. The set of quantum states is convex (Bengtsson and Życzkowski, 2006), and its boundary consists of pure states which in matrix terms are rank one projectors. Convex combinations of pure states lie inside the set and are called mixed states.

3.2.1. Entanglement. Entanglement is one of the most important phenomena in quantum information theory. We say that a state $\rho$ is separable iff it can be written in the following form:

$$
\rho=\sum_{i=1}^{M} q_{i} \rho_{i}^{A} \otimes \rho_{i}^{B},
$$

where $q_{i}>0$ and $\sum_{i=1}^{M} q_{i}=1$. A state that is not separable is called entangled. It is an open problem of great importance and under investigation to decide if a given quantum state is entangled or not.

3.2.2. Subsystems. Given two states $\rho^{A}, \rho^{B}$ of two systems $A$ and $B$, the product state $\rho^{A B}$ of the composed system is obtained by taking the Kronecker product of the states, i.e., $\rho^{A B}=\rho^{A} \otimes \rho^{B}$.

Let $\left[\rho^{A B}\right]_{k l}$ be a matrix representing a quantum system composed of two subsystems of dimensions $M$ and $N$. We want to index the matrix elements of $\rho$ using two double indices $\left[\rho^{A B}\right]_{n \nu}^{m}$, so that Latin indices correspond to the system $A$ and Greek indices correspond to the system $B$. The relation between the indices is $k=(m-1) N+\mu, l=(n-1) N+\nu$. The partial trace with respect to system $B$ reads

$$
\operatorname{tr}_{B}\left(\rho^{A B}\right)=\sum_{\mu} \underset{n \mu}{\rho m \mu}=\rho^{A}
$$

and the partial trace with respect to system $A$ reads

$$
\operatorname{tr}_{A}\left(\rho^{A B}\right)=\sum_{m} \underset{m \nu}{\rho m \mu}=\rho^{B}
$$

Given the state of the composed system $\rho^{A B}$, the state of subsystems can by found by taking the partial trace of $\rho^{A B}$ with respect to one of the subsystems. It should be noted that tracing-out is not a reversible operation, so, in a general case,

$$
\rho^{A B} \neq \operatorname{tr}_{A}\left(\rho^{A B}\right) \otimes \operatorname{tr}_{B}\left(\rho^{A B}\right) .
$$

\subsection{Completely Positive Trace-Preserving (CPTP)} maps. We say that an operation is physical if it transforms density operators into density operators. Additionally, we assume that physical operations are linear. Therefore, in order for an operation $\Phi(\cdot)$ to be physical, it has to fulfil the following set of conditions:

(i) For any operator $\rho$ its image under operation $\Phi$ has to have its trace and positivity preserved, i.e., if $\operatorname{tr}(\rho)=$ $1, \rho \geq 0, \rho=\rho^{\dagger}$, then $\operatorname{tr}(\Phi(\rho))=1, \Phi(\rho) \geq$ $0, \Phi(\rho)=\Phi(\rho)^{\dagger}$.

(ii) The operator $\Phi$ has to be linear:

$$
\Phi\left(\sum_{i} p_{i} \rho_{i}\right)=\sum_{i} p_{i} \Phi\left(\rho_{i}\right) .
$$

(iii) The extension of the operator $\Phi$ to any larger dimension that acts trivially on the extended system has to preserve positivity. This feature is called complete positivity. This means that for all positive semidefinite $\rho, \xi \geq 0$, the following holds:

$$
\left(\Phi \otimes \mathbb{I}_{\operatorname{dim}(\xi)}\right)(\rho \otimes \xi)=\Phi(\rho) \otimes \xi \geq 0 .
$$

CPTP maps are often called quantum channels.

3.3.1. Kraus form. Any operator $\Phi$ that is completely positive and trace preserving can be expressed in the socalled Kraus form (Bengtsson and Życzkowski, 2006), which consists of a finite set $\left\{E_{k}\right\}$ of Kraus operators, i.e., matrices that fulfil the completeness relation: $\sum_{k} E_{k}^{\dagger} E_{k}=\mathbb{I}$. The image of the state $\rho$ under the map $\Phi$ is given by

$$
\Phi(\rho)=\sum_{k} E_{k} \rho E_{k}^{\dagger} .
$$

3.4. Measurement. Quantum states cannot be observed directly. In the literature, two main types of measurements are considered: von Neumann measurements 
and POVM (Positive Operator Valued Measure) measurements. In this paper we use only von Neumann measurements, but for the sake of completeness we also define POVM measurements.

The mathematical formulation of von Neumann measurement is given by a map from a set of projection operators to real numbers.

Let us consider an orthogonal complete set of projection operators $P=\left\{P_{i}\right\}_{i=1}^{N}$ and the set of real measurement outcomes $O=\left\{o_{i}\right\}_{i=1}^{N}$. The mapping $P \rightarrow O$ is called the von Neumann measurement. Assuming the system is in the state $\rho$, the probability $p_{i}$ of measuring outcome $o_{i}$ is given by the relation $p_{i}=\operatorname{tr}\left(P_{i} \rho\right)$.

The POVM measurement can be considered a generalisation of the von Neumann measurement. Let us take a set of positive operators $F=\left\{F_{i}\right\}_{i=1}^{N}$ such that $\sum_{i=1}^{N} F_{i}=\mathbb{I}$ and the set of real measurement outcomes $O=\left\{o_{i}\right\}_{i=1}^{N}$. The mapping $F \rightarrow O$ is called the POVM measurement. Given the system in the state $\rho$, the probability $p_{i}$ of measuring the outcome $o_{i}$ is given by the relation $p_{i}=\operatorname{tr}\left(F_{i} \rho\right)$.

\section{Overview of Grover's algorithm}

Grover's unordered database search algorithm is one of the most important quantum algorithms. This is due to the fact that many algorithmic problems can be reduced to exhaustive search.

The main idea of the algorithm is to amplify the probability of the state which represents the sought element. The algorithm is probabilistic and may fail to return the proper result. Fortunately, the probability of success is reasonably high.

4.1. Problem. Let $X$ be a set and let $f: X \rightarrow\{0,1\}$, such that

$$
f(x)=\left\{\begin{array}{lll}
1 & \text { if } & x=x_{0} \\
0 & \text { if } & x \neq x_{0}
\end{array}\right.
$$

$x \in X$, for some marked $x_{0} \in X$.

For simplicity, we assume that $X$ is a set of binary strings of length $n$. Therefore, $|X|=2^{n}$ and $f:\{0,1\}^{n} \rightarrow\{0,1\}$. We can map the set $X$ to a set of states over $\mathbb{C}^{\otimes 2^{n}}$ in a natural way: $x \leftrightarrow|x\rangle$, forming an a orthogonal, complete set of vectors. The goal of the algorithm is to find the marked element.

4.2. Algorithm. Grover's algorithm is composed of two main procedures: the oracle and diffusion.

4.2.1. Oracle. By an oracle we mean a function that marks one defined element. In the case of Grover's algorithm, the marking of the element is done by the negation of the amplitude of the sought state.
With the use of elementary quantum gates the oracle can be constructed using ancilla $|q\rangle$ in the following way:

$$
O|x\rangle|q\rangle=|x\rangle|q \oplus f(x)\rangle,
$$

where $\oplus$ denotes addition modulo 2 . If the register $|q\rangle$ is prepared in the state

$$
|q\rangle=H|1\rangle=\frac{|0\rangle-|1\rangle}{\sqrt{2}},
$$

where $H$ denotes the Hadamard gate, then, by substitution, Eqn. (7) can be written as

$$
O|x\rangle \frac{|0\rangle-|1\rangle}{\sqrt{2}}=(-1)^{f(x)}|x\rangle \frac{|0\rangle-|1\rangle}{\sqrt{2}} .
$$

By tracing out the ancilla, we obtain

$$
O|x\rangle=-(-1)^{f(x)}|x\rangle
$$

4.2.2. Diffusion. The operator $D$ rotates any state around the state

$$
|\psi\rangle=\frac{1}{\sqrt{2^{n}}} \sum_{x=0}^{2^{n}-1}|x\rangle,
$$

where $D$ can be written as

$$
D=-H^{\otimes n}(2|0\rangle\langle 0|-\mathbb{I}) H^{\otimes n}=2|\psi\rangle\langle\psi|-\mathbb{I} .
$$

4.2.3. Initialisation. We begin in the ground state $|0 \ldots 00\rangle$. In the first step of the algorithm we apply the Hadamard gate $H^{\otimes n}$ to the entire register. This transforms the initial state into flat superposition of computational base states:

$$
H^{\otimes n}|0 \ldots 0\rangle=\frac{1}{\sqrt{n}}(|0 \ldots 00\rangle+\cdots+|1 \ldots 11\rangle) .
$$

4.2.4. Grover iteration. The core of the algorithm consists of the applications of the so-called Grover iteration gate $G=D \cdot O$. This procedure causes the sought state to be amplified and other states to be attenuated.

4.2.5. Number of iterations. The application of the diffusion operator to the base state $|x\rangle$ gives

$$
D|x\rangle=-\left|x_{0}\right\rangle+\frac{2}{N} \sum_{y}|y\rangle .
$$

The application of this operator on any state gives

$$
\begin{aligned}
D|x\rangle & =\sum_{i} \alpha_{i}\left(-|x\rangle+\frac{2}{N} y \sum_{y}|y\rangle\right) \\
& =\sum_{i}\left(-\alpha_{i}+2 s\right)|x\rangle,
\end{aligned}
$$


where

$$
s=\frac{1}{N} \sum_{i} \alpha_{i}
$$

A $k$-fold application of Grover's iteration $G$ to the initial state $|s\rangle$ leads to (Bouwmeester et al., 2000; Bugajski, 2001)

$$
G^{k}|s\rangle=\alpha_{k} \sum_{x \neq x_{0}}|x\rangle+\beta_{k}\left|x_{0}\right\rangle
$$

with real coefficients

$$
\alpha_{k}=\frac{1}{\sqrt{N-1}} \cos (2 k+1) \theta, \quad \beta_{k}=\sin (2 k+1) \theta,
$$

where $\theta$ is an angle that fulfils the relation

$$
\sin (\theta)=\frac{1}{\sqrt{N}}
$$

Therefore the coefficients $\alpha_{k}, \beta_{k}$ are periodic functions of $k$. After a series of iterations, $\beta_{k}$ rises. The influence of the marked state $\left|x_{0}\right\rangle$ on the state of the register results in the evolution of the initial state $|s\rangle$ towards the marked state.

$\beta_{k}$ attains its maximum after approximately $\frac{\pi}{4} \sqrt{N}$ steps. The number of steps needed to transfer the initial state towards the marked state is of order $O(\sqrt{N})$. In the classical case the number of steps is of order $O(N)$.

4.2.6. Measurement. The last step of Grover's algorithm is a von Neumann measurement. The probability of obtaining the proper result is $\left|\beta_{k}\right|^{2}$.

\section{Noise model}

The above discussion of the quantum search algorithm has been conducted using the state vector formalism. In order to incorporate noise into the quantum computation model, we have to make use of density operators which define the quantum state in the most general way.

5.1. Quantum noise. Microscopic systems that are governed by the laws of quantum mechanics are hard to control and, at the same time, to separate from the environment. The interaction with the environment introduces noise into the quantum system. Therefore any future quantum computer will also be prone to noise.

One-qubit noise. There are several one-parameter families of one-qubit noisy channels that are typically discussed in the literature (Nielsen and Chuang, 1999). We present them briefly below.

Depolarising channel. This is a bi-stochastic channel that transforms any state into a maximally mixed state with a given probability $\alpha$. The family of channels can be defined using a four-element set of Kraus operators

$$
\left\{\sqrt{1-\alpha} \mathbb{I}, \sqrt{\frac{\alpha}{3}} \sigma_{x}, \sqrt{\frac{\alpha}{3}} \sigma_{y}, \sqrt{\frac{\alpha}{3}} \sigma_{z}\right\},
$$

where

$$
\begin{aligned}
\mathbb{I} & =\left[\begin{array}{ll}
1 & 0 \\
0 & 1
\end{array}\right], & \sigma_{x} & =\left[\begin{array}{ll}
0 & 1 \\
1 & 0
\end{array}\right], \\
\sigma_{y} & =\left[\begin{array}{ll}
0 & -i \\
i & 0
\end{array}\right], & \sigma_{z} & =\left[\begin{array}{ll}
1 & 0 \\
0 & -1
\end{array}\right]
\end{aligned}
$$

are Pauli matrices.

Amplitude damping. The amplitude damping channel transforms $|1\rangle$ into $|0\rangle$ with a given probability $\alpha$. The state $|0\rangle$ remains unchanged. The set of Kraus operators is

$$
\left\{\left[\begin{array}{cc}
1 & 0 \\
0 & \sqrt{1-\alpha}
\end{array}\right],\left[\begin{array}{cc}
0 & \sqrt{\alpha} \\
0 & 0
\end{array}\right]\right\}
$$

Phase damping. Phase damping in a quantum phenomenon describes the loss of quantum information without the loss of energy. It is described by the following set of Kraus operators:

$$
\left\{\left[\begin{array}{cc}
1 & 0 \\
0 & \sqrt{1-\alpha}
\end{array}\right],\left[\begin{array}{cc}
0 & 0 \\
0 & \sqrt{\alpha}
\end{array}\right]\right\}
$$

Bit flip. The bit flip family of channels is the quantum version of the classical binary symmetric channel. The action of the channel might be interpreted in the following way: it flips the state of a qubit from $|0\rangle$ to $|1\rangle$ and from $|1\rangle$ to $|0\rangle$ with probability $\alpha$. Kraus operators for this family of channels consist of a matrix proportional to the identity and a matrix proportional to the negation gate,

$$
\left\{\sqrt{1-\alpha} \mathbb{I}, \sqrt{\alpha} \sigma_{x}\right\}
$$

Phase flip. The phase flip channel acts similarly to the bit flip channel with the distinction that a $\sigma_{z}$ gate is applied randomly to the qubit

$$
\left\{\sqrt{1-\alpha} \mathbb{I}, \sqrt{\alpha} \sigma_{z}\right\}
$$

Bit-phase flip. The bit-phase flip channel may be considered a joint application of bit and phase flip gates to a qubit. Its Kraus operators are as follows:

$$
\left\{\sqrt{1-\alpha} \mathbb{I}, \sqrt{\alpha} \sigma_{y}\right\}
$$

In all of the above families of channels, the real parameter $\alpha \in[0,1]$ can be interpreted as the amount of noise introduced by the channel. 


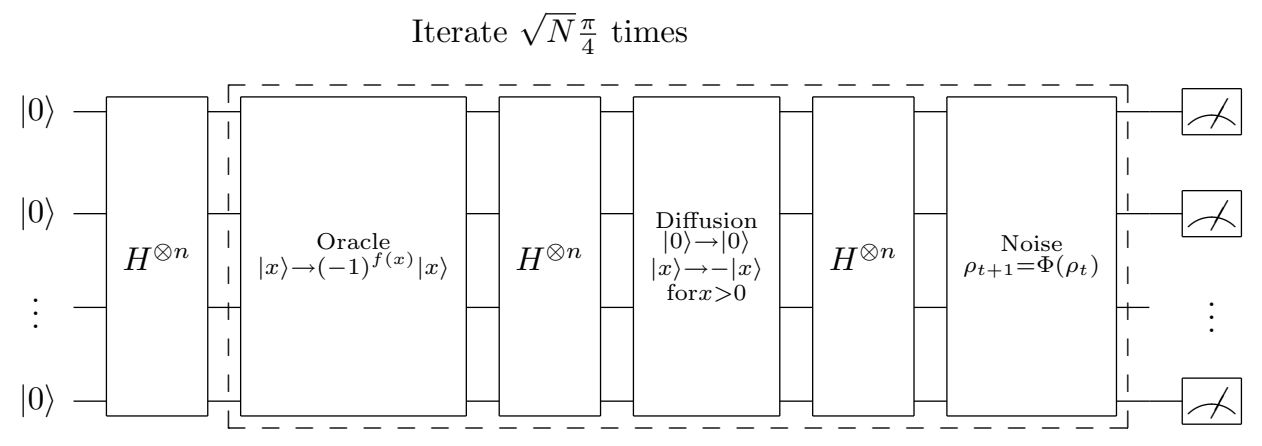

Fig. 1. Circuit for Grover's algorithm extended with a non-unitary noisy channel.

Multiqubit local channels. Our goal is to extend the noise acting on distinct qubits to the entire registers. We assume that the appearance of an error on a given qubit is independent of an error appearing on any other qubits.

In order to apply noise operators to multiple qubits, we form a new set of Kraus operators acting on a larger Hilbert space.

We assume that we have the set of $n$ one-qubit Kraus operators $\left\{e_{k}\right\}_{k=1}^{n}$. We construct the new set of $n^{N}$ operators $\left\{E_{k}\right\}_{k=1}^{n^{N}}$ that act on a Hilbert space of dimension $2^{N}$ by applying the following formula:

$$
\left\{E_{k}\right\}=\bigcup_{I}\left\{e_{i_{1}} \otimes e_{i_{2}} \otimes \ldots \otimes e_{i_{N}}\right\},
$$

where

$$
I=\left\{i_{1}\right\}_{i_{1}=1}^{n} \times\left\{i_{2}\right\}_{i_{2}=1}^{n} \times \ldots \times\left\{i_{N}\right\}_{i_{N}=1}^{n} .
$$

One should note that the extended channel $\Phi(\rho)=$ $\sum_{k} E_{k} \rho E_{k}^{\dagger}$ is by definition local (Bengtsson and $\dot{\mathrm{Z} y}$ czkowski, 2006).

By applying Eqn. (19) to the sets of operators listed above, we obtain one-parameter families of local noisy channels, which we use in further investigations.

5.2. Application of noise to the algorithm. In order to simulate noisy behaviour of the system implementing the algorithm, we apply a noisy channel after every Grover iteration. The evolution of the system is described by the following procedure, which is graphically depicted in Fig. 1 .

1. Prepare the system in state $\rho_{0}:=\left|0^{\otimes n}\right\rangle\left\langle 0^{\otimes n}\right|$.

2. $\rho:=H^{\otimes n} \rho_{0} H^{\otimes n \dagger}$

3. $\left\lfloor\frac{\pi}{4} \sqrt{N}\right\rfloor$ times do:

(a) apply Grover's iteration $\rho:=G \rho G^{\dagger}$,

(b) apply noise $\rho:=\Phi(\rho)$.
4. Perform an orthogonal measurement in the computational basis. The probability of finding the sought element $\xi$ is $p=\langle\xi|\rho| \xi\rangle$.

This approach simplifies the physical reality, but it is sufficient to study the robustness of the algorithm in the presence of noise. In order to study the discussed problem, we make use of numerical simulations. Therefore some simplification is necessary as the size of the problem grows exponentially fast with the number of qubits.

The tool we use is quantum-octave (Gawron et al., 2010), a library that contains functions for simulation and analysis of quantum processes.

In our model we assume that it is easy to verify the correctness of the quantum search algorithm. It is an assumption usually made in the complexity analysis of search algorithms.

\section{Analysis of the influence of noise on the efficiency of the algorithm}

An interesting question arises: "What is the maximal amount of noise for which Grover's algorithm is more efficient than any classical search algorithm?"

Grover's algorithm is probabilistic, therefore we cannot expect to obtain a valid outcome with certainty. We assume that if the algorithm fails in a given run we will rerun it. There is a certain number of reruns for which the quantum algorithm is worse than the classical. We are interested only in the statistical behaviour of algorithm and calculate the mean value of repetitions.

Let $k=\left\lfloor\frac{N}{2} / \frac{\pi}{4} \sqrt{N}\right\rfloor$ be the maximal number of single runs of Grover's algorithm for which quantum search is faster than the classical one.

We compute the minimal value of success probability $p_{\text {min }}$ of a single run of Grover's algorithm for which we obtain a valid result with confidence $C$,

$$
p_{\min }=\min _{p}\left\{1-(1-p)^{k} \geq C\right\} .
$$

Numerically obtained values of $p_{\min }$ for the confidence level $C=0.95$ for Grover's algorithm are listed in Table 1. 
Table 1. Values of $k$ and $p_{\text {min }}$ for Grover's algorithm.

\begin{tabular}{|c|r|c|}
\hline Size of the system & \multicolumn{1}{|c|}{$\mathrm{k}$} & $p_{\min }$ \\
\hline$N=2^{3}$ & 1 & 0.95000 \\
$N=2^{4}$ & 2 & 0.77639 \\
$N=2^{5}$ & 3 & 0.63160 \\
$N=2^{6}$ & 5 & 0.45072 \\
$N=2^{7}$ & 7 & 0.34816 \\
$N=2^{8}$ & 10 & 0.25887 \\
\hline
\end{tabular}

For our numerical experiment we assume that the sought element $\xi$ lies in the "middle" of the space of elements, i.e., $\xi=2^{n-1}$.

Plots in Fig. 2 depict the influence of the noise parameter $\alpha$ on a successful run of Grover's algorithm acting on six qubits. These values of the parameter $\alpha$ for which the plots are above the threshold level $p_{\min }$ can be considered the amounts of noise which do not make the quantum search algorithm less efficient than the classical search algorithms.

We can compare the probabilities from plots in Fig. 2 and these for other sizes of quantum registers with $p_{\text {min }}$ and find the value of the noise parameter $\alpha$ for which it is equal to $p_{\min }$. The results of the comparison are collected in Table 2 for the confidence level $C=0.95$ and for the channels described in Section 5

Table 2. Maximal values of the noise parameter $\alpha$ for which Grover's search algorithm is as efficient as the classical search algorithm in terms of the number of uses of the oracle.

\begin{tabular}{|l|l|l|l|}
\hline$C=0.95$ & depolarising & amplitude damping & phase damping \\
\hline$N=2^{4}$ & 0.025 & 0.069 & 0.177 \\
$N=2^{5}$ & 0.032 & 0.010 & 0.204 \\
$N=2^{6}$ & 0.031 & 0.104 & 0.190 \\
$N=2^{7}$ & 0.026 & 0.094 & 0.158 \\
$N=2^{8}$ & 0.020 & 0.075 & 0.122 \\
\hline \hline & bit flip & phase flip & bit-phase flip \\
\hline$N=2^{4}$ & 0.025 & 0.047 & 0.018 \\
$N=2^{5}$ & 0.032 & 0.054 & 0.024 \\
$N=2^{6}$ & 0.031 & 0.050 & 0.023 \\
$N=2^{7}$ & 0.026 & 0.041 & 0.020 \\
$N=2^{8}$ & 0.020 & 0.031 & 0.015 \\
\hline
\end{tabular}

In the case of three qubits we have found that, if we expect a confidence level $C=0.95$ or higher, Grover's algorithm is never better than the classical search algorithm. This means that if we want to get the result with high probability, we have to repeat the quantum search so many times that it is more efficient to perform this task classically.

In other cases we have obtained the values of the noise parameter $\alpha$ between $\sim 0.010$ and $\sim 0.2$ depending on the noise type and the system size. We observe that, even if the amount of noise is larger in bigger systems (which causes the algorithm to be less efficient), the noise is compensated by the quantum speed-up.

The results gathered in Table 2 do not form a monotonic pattern. To understand this fact, we have to take into account that two factors influence these numbers. The first one is due to the fact that the same value of the noise parameter $\alpha$ has greater influence on the quantum system for bigger numbers of qubits and for larger $N$ the number of Grover iterations and noisy channel applications $k$ is increasing. At the same time, the more qubits used to perform the search algorithm, the more important the quantum speed-up.

\section{Summary}

In this work we have shown a new way of analysing the influence of quantum noise on the quantum search algorithm. Our method uses the model of density matrices and quantum channels represented in the Kraus form.

We can conclude that the simulations and analysis have shown that it is only for small amounts of noise that the quantum search algorithm is still more efficient than any classical algorithm.

From our numerical results we conclude that different forms of noise have different impact on the efficiency of the quantum search algorithm. The least destructive form of noise is phase damping, more destructive is amplitude damping, and the most destructive is the depolarizing channel.

Further work would have to take into account quantum error correcting codes and more precise noise models dependent on the implementation. One of the research directions would be to analyse the quantum search algorithm in the framework of control Hamiltonians taking into account Markovian approximation of quantum noise.

\section{Acknowledgment}

We acknowledge the financial support by the Polish Ministry of Science and Higher Education (MNiSzW) under the grants N N519 442339 and N N516 481840. The work of Piotr Gawron was partially supported by the MNiSW project IP2010 009770. The numerical calculations presented in this work were performed on the Leming server of the Institute of Theoretical and Applied Informatics, Polish Academy of Sciences.

\section{References}

Azuma, H. (2005). Higher-order perturbation theory for decoherence in Grover's algorithm, Physical Review A 72(4): 42305.

Barnes, J.P. and Warren, W.S. (1999). Decoherence and programmable quantum computation, Physical Review A 60(6): 4363-4374. 

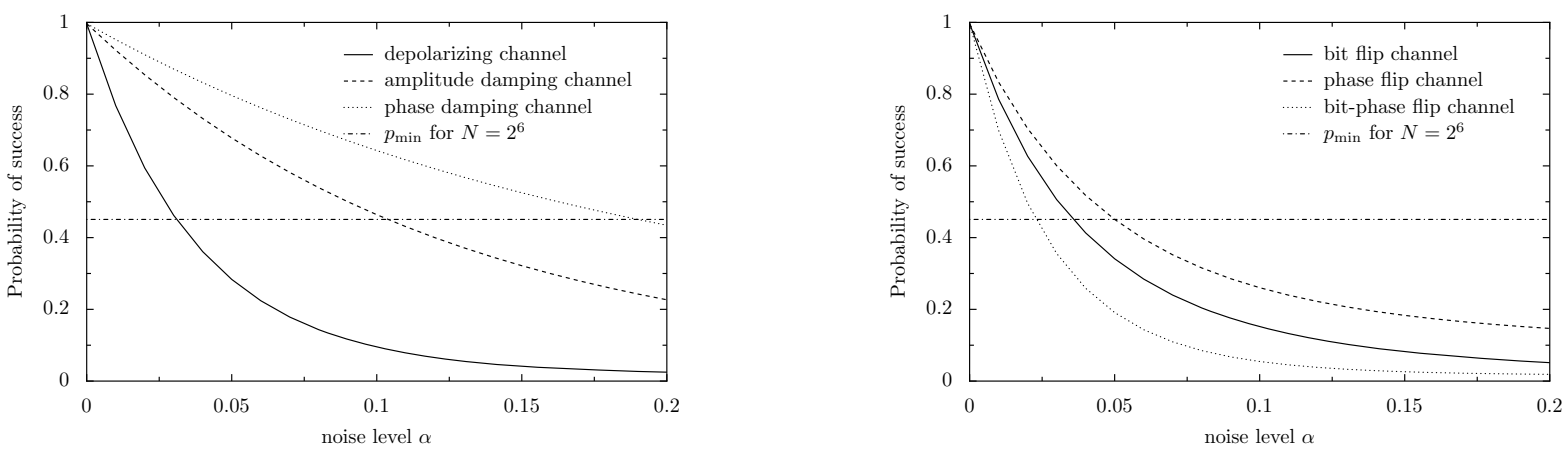

Fig. 2. Probabilities of a successful run of Grover's algorithm as a function of the noise parameter $\alpha$ : case of six qubits. The value for which the plots attain the threshold $p_{\min }$ is shown in Table1

Bengtsson, I. and Życzkowski, K. (2006). Geometry of Quantum States. An Introduction to Quantum Entanglement, Cambridge University Press, Cambridge.

Bouwmeester, D., Ekert, A. and Zeilinger, A. (2000). The Physics of Quantum Information: Quantum Cryptography, Quantum Teleportation, Quantum Computation, Physics and Astronomy Online Library, Springer, http: / /www.springer.com/physics/quantum +physics/book/978-3-540-66778-0

Bugajski, S. (2001). Quantum search, Archiwum Informatyki Teoretycznej i Stosowanej 13(2): 143-150.

Gawron, P., Klamka, J., Miszczak, J.A. and Winiarczyk, R. (2010). Extending scientific computing system with structural quantum programming capabilities, Bulletin of the Polish Academy of Sciences: Technical Sciences 58(1): 7788.

Grover, L. (1996). A fast quantum mechanical algorithm for database search, Proceedings of the 28th Annual ACM Symposium on the Theory of Computation, Philadelphia, PA, USA, pp. 212-219.

Grover, L.K. (1997). Quantum mechanics helps in searching for a needle in a haystack, Physical Review Letters 79(2): 325.

Grover, L.K. (1998). A framework for fast quantum mechanical algorithms, Proceedings of the 30th Annual ACM Symposium on Theory of Computing (STOC), Dallas, TX, USA, pp. 53-62.

Konstadakis, C. and Ellinas, D. (2001). Noisy Grover's Searching Algorithm, OSA Technical Digest Series, Optical Society of America, Rochester/New York, NY.

Long, G.L., Li, Y.S., Zhang, W.L. and Tu, C.C. (2000). Dominant gate imperfection in Grover's quantum search algorithm, Physical Review A 61(4): 42305.

Nielsen, M. and Chuang, I. (1999). Quantum Computation and Quantum Information, Cambridge University Press, Cambridge.

Pablo-Norman, B. and Ruiz-Altaba, M. (1999). Noise in Grover's quantum search algorithm, Physical Review A 61(1): 12301.

Salas, P.J. (2008). Noise effect on Grover algorithm, The European Physical Journal D 46(2): 365-373.
Shapira, D., Mozes, S. and Biham, O. (2003). Effect of unitary noise on Grover's quantum search algorithm, Physical Review A 67(4): 42301.

Shenvi, N., Brown, K.R. and Whaley, K.B. (2003). Effects of a random noisy oracle on search algorithm complexity, Physical Review A 68(5): 52313.

Zhirov, O.V. and Shepelyansky, D.L. (2006). Dissipative decoherence in the Grover algorithm, The European Physical Journal D 38(2): 405-408.

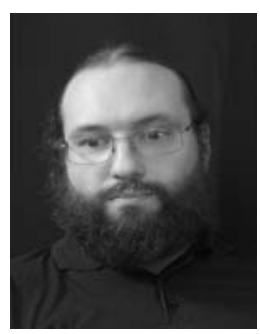

Piotr Gawron, Ph.D., Eng., works in the Quantum Systems of Informatics Group of the Institute of Theoretical and Applied Informatics, Polish Academy of Sciences. His main areas of research include noise in quantum processes, quantum games, and simulation of quantum processes.

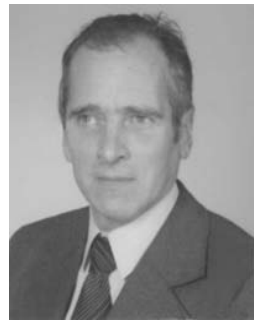

Jerzy Klamka, Ph.D., Prof., is a full member of the Polish Academy of Sciences and works in the Quantum Systems of Informatics Group of the Institute of Theoretical and Applied Informatics, Polish Academy of Sciences. The main areas of his research include controllability and observability of linear and non-linear dynamical systems, as well as mathematical foundations of quantum computations. He is the author of numerous monographs and many papers published in international journals.

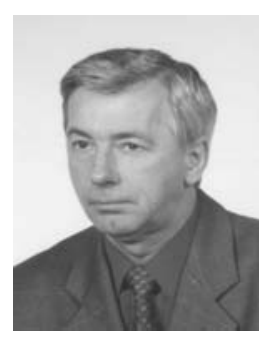

Ryszard Winiarczyk, Ph.D., Eng., received the M.Sc and Ph.D. degrees from the Silesian University of Technology in Gliwice. In 2001, he was appointed the head of the Quantum System of Informatics Group in the Institute of Theoretical and Applied Informatics, Polish Academy of Sciences. His main research interest in the quantum informatics domain is the development of software environment for quantum computations.

Received: 3 March 2011

Revised: 10 August 2011 